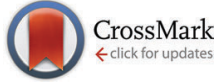

Cite this: J. Mater. Chem. B, 2015, 3, 9269

\title{
Macromolecular crowding and hydrophobic effects on Fmoc-diphenylalanine hydrogel formation in PEG : water mixtures $\dagger$
}

\begin{abstract}
Md. Musfizur Hassan, Adam D. Martin and Pall Thordarson*
A small peptide-based gelator forms stable hydrogels in an aqueous mixture with a range of poly(ethylene glycol) PEGs, from the ethylene glycol monomer to PEG 20000 with stronger gels forming in polymeric PEG. Spectroscopic studies on these systems reveal significant secondary structural changes when compared to gels formed from pure water. The use of PEG also facilitates the incorporation and controlled release of poorly water-soluble anti-cancer drugs such as Temozolomide and Paclitaxel $\left(\right.$ Taxol $\left.{ }^{\circledR}\right)$. This work provides a powerful insight into the role of macromolecular crowding and hydrophobic interactions in not only hydrogels formed from small molecules but potentially also biological gel-like materials such as the cytosol and the extracellular matrix (ECM).
\end{abstract}

Received 14th October 2015, Accepted 5th November 2015 DOI: $10.1039 / c 5 t b 02139 a$

www.rsc.org/MaterialsB mixing polymers with self-assembled gels. ${ }^{13-17}$ In all of these cases, the polymer or polymer/water mixture is added after solubilising the gelators and in most cases, the addition of polymer actually appears to make the resulting gel softer. An exception to this is the work of Yang and co-workers who showed that mixing peptide gelator-type molecules with agarose resulted in stronger gels. ${ }^{13}$ However, it is worth noting that the agarose used in that study forms fairly strong gels by itself $\left(G^{\prime}=9 \mathrm{kPa}\right)$.

Self-assembled hydrogels also have recently attracted interest due to promising potential applications in the fields of medicine, ${ }^{18-20}$ including as cell scaffolds ${ }^{21,22}$ and in drug delivery, ${ }^{15,23,24}$ however, in the latter case the range of drugs that can be used is somewhat restricted by solubility and stability issues. In the pharmaceutical industry, polyethylene glycol (PEG) is often used to enhance solubility but PEGs are also frequently used to create macromolecular crowding conditions in vitro. ${ }^{2,25-28}$

Here we show the formation of self-assembled gels from the gelator 9-fluorenylmethyloxycarbonyl-diphenylalanine $e^{29-31}$ (Fmoc-PhePhe) 1 (Chart 1) in various PEG : water mixtures, including ethylene glycol, with the PEG/water ratio (v/v), molecular weight (from ethylene glycol to PEG 20000) of the PEG and the order of addition (gelator to PEG or water first) all influencing the resulting gel properties. The change in gelation behaviour depending on the concentration and molecular weight of the PEG used may be explained by a combination of macromolecular crowding and hydrophobic effects. We also show that otherwise poorly soluble drugs like Paclitaxel (Taxol ${ }^{\mathbb{R}}$ ) and Temozolomide can be released effectively from gels based on 1 in PEG : water. 
<smiles>O=C(N[C@@H](Cc1ccccc1)C(=O)N[C@@H](Cc1ccccc1)C(=O)O)OCC1c2ccccc2-c2ccccc21</smiles><smiles>CC(O)CCOC(C)C</smiles>

Ethylene glycol: $n=0$

PEG 200: $\quad n$ ca. 4-5

PEG 400: $\quad n$ ca. $8-9$

PEG 8,000: $\quad n$ ca. 180

PEG 10,000: $\quad n$ ca. 230

PEG 20,000: $\quad n$ ca 460

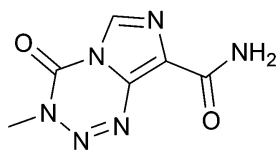

Temozolomide

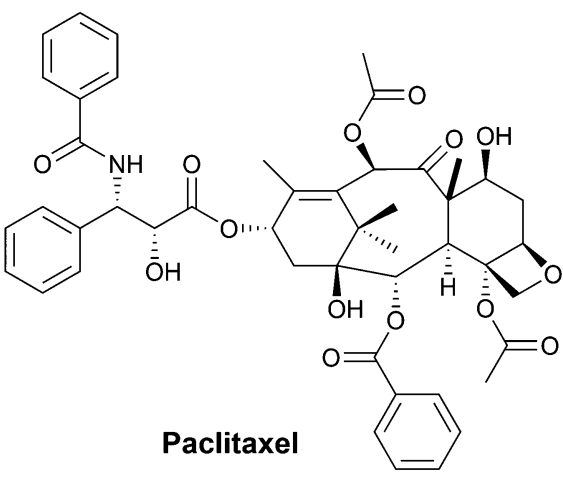

Chart 1

\section{Results and discussions}

\section{Gel preparation and rheology}

In our standard protocol, gels were prepared by dissolving $\mathbf{1}$ in the chosen PEG; at room temperature for liquid PEGs and at $70{ }^{\circ} \mathrm{C}$ for high molecular weight solid PEGs, followed by the addition of milliQ water, with a gel forming over a short timescale under ambient conditions. Using this methodology, we explored the gelation behaviour of $\mathbf{1}$ in a range of different molecular weight PEGs, ranging from the monomer repeat unit of PEG; ethylene glycol, to PEG $20000\left(M_{\mathrm{n}} \approx 20000\right)$. Of the PEGs tested (Fig. 1 - filled circles and squares, Fig. S1-S6, ESI $\dagger$ ), ethylene glycol, PEG 200 and PEG 400 are liquids at room temperature, with the rest being solids. Using $1 \%(\mathrm{w} / \mathrm{v}) 1$ in a mixture of 50\% PEG and 50\% water (v/v), we observed gelation for all of the PEGs tested. Furthermore, the storage modulus $G^{\prime}$ (often used as an indicator of gel strength) stays much the same (approximately $15 \mathrm{kPa}$ ) for PEGs from PEG 400 up to PEG 10000. PEG 20000 forms slightly stronger gels $\left(G^{\prime}=30 \mathrm{kPa}\right)$, whereas weak gels are formed using ethylene glycol $\left(G^{\prime}=140 \mathrm{~Pa}\right)$ or PEG $200\left(G^{\prime}=1.6 \mathrm{kPa}\right)$. The large plateau seen in Fig. 1 is evidence that for a range of PEGs, there appears to be no correlation between the molecular weight of the polymer and gel strength, which is comparable (Fig. 1 - right) if not stronger than gels formed from 1 using a pH switch method. ${ }^{32}$ Macroscopically, the
- With $1 \%(w / v)$ 1: Storage Modulus (G')

- With $1 \%(w / v)$ 1: Loss Modulus (G")

- Without 1: Storage Modulus (G')

- Without 1: Loss Modulus (G')

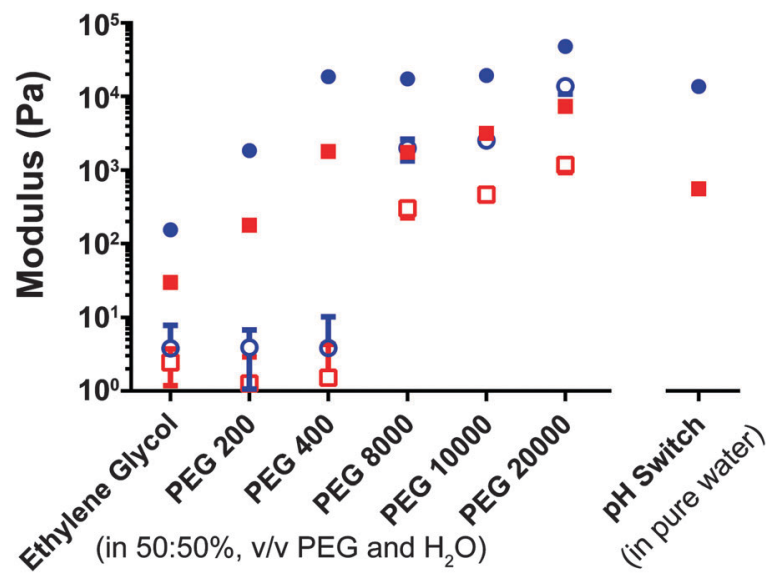

Fig. 1 Storage $\left(G^{\prime}\right.$ - blue circle markers) and loss modulus ( $G^{\prime \prime}$ red circle markers) of different molecular weight PEGs in water $(50: 50 \%, \mathrm{v} / \mathrm{v})$, recorded at a frequency of $1 \mathrm{~Hz}$ and strain of $1 \%$ both with $1 \%(\mathrm{w} / \mathrm{v}) 1$ (filled markers) and without any gelator 1 present (hollow markers). A gel of 1 formed via the $\mathrm{pH}$ switch method is also shown to right. Error bars indicate two times the log standard deviation from repeat experiments $(n=3)$.

gels appear fairly homogeneous but they also vary from nearly transparent for PEG 200 : water mixtures to completely opaque for PEG 20000 : water mixtures (Fig. S7, ESI $\dagger$ ).

In the absence of $1,50: 50 \%(\mathrm{v} / \mathrm{v})$ mixtures of ethylene glycol, PEG 200 and PEG 400 are homogenous fluid solutions with $G^{\prime}<20$ Pa (Fig. 1b and Fig. S8-S13, ESI $\dagger$ ) at all PEG: water ratios. The higher molecular weight PEG 8000, PEG 10000 and PEG 20000 gradually form gel-like solids at room temperature with $50: 50 \%(\mathrm{v} / \mathrm{v})$ water as shown in Fig. 1 (open circles \& squares) that are approximately an order of magnitude weaker than the corresponding gels formed with 1 present.

Adams and co-workers noted in their work on adding concentrated DMSO solutions of 1 and another related naphthalenecapped peptide gelator, to solutions of water and dextran, that the $G^{\prime}$ and $G^{\prime \prime}$ of the resulting gelator appeared to show a negative correlation with the viscosity of the water/polymer mixtures. ${ }^{16}$ In our case, the viscosity of the PEG/water mixtures change when transitioning from a liquid PEG to solid PEG (see Fig. S14, ESI $\dagger$ ), however, the $G^{\prime}$ and $G^{\prime \prime}$ values shown in Fig. 1 do not follow this same trend, instead these values remain similar from PEG 400 to PEG 20000 , indicating that the storage modulus of these hydrogels cannot be correlated with PEG viscosity.

Although 1 does not form a strong gel in 50:50\% (v/v) ethylene glycol:water, the fact a gel is formed suggests that hydrophobic interactions also play a significant role in these systems. Given that PEG 400, 8000 and 10000 all yield hydrogels of similar rheological properties, PEG 400 was selected as the PEG of choice for further investigations. PEG 400 is widely used as an excipient in pharmaceutical formulations, partly due to its favourable safety profile. ${ }^{33}$ 
a)

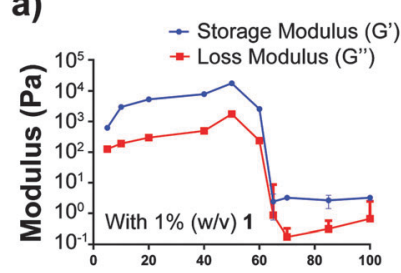

Ratio (\%, v/v) PEG 400:water b)

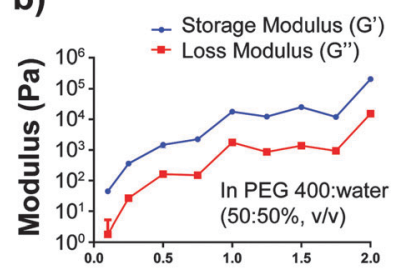

Concentration $1(\%, w / v)$

Fig. 2 Storage $\left(G^{\prime}\right)$ and loss modulus $\left(G^{\prime \prime}\right)$ recorded at a frequency of $1 \mathrm{~Hz}$ and strain of $1 \%$ of 1 in PEG 400 : water. (a) With $1 \%(\mathrm{w} / \mathrm{v}) 1$ with different ratio's ( $v / v)$ of PEG 400 : water. (b) With a range of concentrations of $1(w / v)$ in PEG 400 : water $(50: 50 \%, v / v)$. Error bars indicate two times the log standard deviation from repeat experiments $(n=3)$.

We next investigated the effect of varying the ratio of PEG 400 : water and the concentrations of 1 (Fig. 2). In the former case, it can be seen that with $1 \%(\mathrm{w} / \mathrm{v}) 1$, the ratio of PEG-towater plays a major role in determining the properties of the gel obtained (Fig. 2a). At concentrations up to approximately $60 \%(\mathrm{v} / \mathrm{v})$ PEG 400 , a gel is formed when water is added, however, at values above $60 \%(\mathrm{v} / \mathrm{v})$ PEG, a liquid is obtained as evident from macroscopic observations of these mixture by the inversion test (Fig. S15, ESI $\dagger$ ), where the least opaque gels appear to form around a $50-60 \%$ PEG $400:$ water $(\mathrm{v} / \mathrm{v})$ ratio. A variation in storage modulus can also be seen for the hydrogels formed, with $5 \%(\mathrm{v} / \mathrm{v})$ PEG 400 in water resulting in a relatively weak gel $\left(G^{\prime}=500 \mathrm{~Pa}\right)$, whilst values for $G^{\prime}$ at $10 \%, 20 \%$ and $40 \%$ PEG 400 in water $(\mathrm{v} / \mathrm{v})$ are very similar. However, a mixture containing $50 \%(\mathrm{v} / \mathrm{v})$ PEG 400 results in a storage modulus that is almost three times higher than that recorded for $40 \%(\mathrm{v} / \mathrm{v})$ PEG 400 as shown in Fig. 2a. As above, the measured $G^{\prime}$ and $\mathrm{G}^{\prime \prime}$ did not appear to have any correlation with measured viscosity of the PEG 400 : water mixtures of different ratios (Fig. S16, ESI†).

Having shown (Fig. 2a) that a 50:50 ratio of PEG: water yields the strongest gels of 1 , we measured the variations in rheological properties of 1 as a function of concentration, from 0.1 to $2 \%$ (w/v) 1, in 50:50 (v/v) PEG 400 : water (Fig. 2b). This data shows a good correlation between the concentration of 1 and the stiffness of the resulting gels. This was expected, however there is a plateau in hydrogel strength at concentrations of 1 between 1 and $1.75 \%(w / v)$. Combined with previous results, this suggests that a $1 \% \mathrm{w} / \mathrm{v}$ of 1 in $50: 50 \%(\mathrm{v} / \mathrm{v})$ PEG 400 : water is the optimal system in terms of stability for gels formed from 1 in PEG 400 : water. Additional rheological thermal stability studies (Fig. S17, ESI $\dagger$ ) on gels from $1 \% \mathrm{w} / \mathrm{v}$ of 1 in 50:50\% (v/v) PEG 400 : water showed that these gels are stable to $60{ }^{\circ} \mathrm{C}$, with total collapse occurring at $90{ }^{\circ} \mathrm{C}$. Comparison of the strain sweeps for the gel formed by the $\mathrm{pH}$ switch method versus gels formed from PEG 400 : water $(50: 50 \%$, v/v) shows that while the crossover points are slightly different (at a slightly lower strain for PEG 400 : water) the linear viscoelastic regions are very similar (Fig. S18 and S19, ESI †).

The sudden increase in storage modulus with as little as 5-10\% (v/v) PEG 400:water appears to be due to solvent crowding effects. Previously it has been shown that mixtures containing similar ratios of PEG 200 and water can stabilise

DNA G-quadruplex structures. ${ }^{26}$ A combination of NMR and dynamic light scattering (DLS) studies performed on a mixture of water and PEG 6000 have concluded that there exists a network of interactions (hydrogen bonds) between the solute and the solvent, which results in the self-assembly of PEG into a more ordered, dehydrated structure and clusters of PEG with diameters from $40-90 \mathrm{~nm} \cdot{ }^{34}$ Interestingly, the $T_{1}$ NMR relaxation measurements on both water and PEG 6000 suggested also that the viscosity of these PEG 6000: water mixtures reached a maximum at about a 50:50 ratio before decreasing again (corresponding to an increase in $T_{1}$ ) with higher PEG 6000 ratios. This is not to imply that the network observed for the high-molecular weight PEG 6000 : water mixtures exists in our work, but rather that there appears to be an optimal ratio for PEG-to-water interactions which may explain the apparent maximum around 50-60\% PEG 400 in Fig. 2.

We envision that similar macromolecular crowding effects as discussed above are responsible for triggering gelation of 1 in PEG-water mixtures, possibly by stabilising gelator hydrogen bonding interactions, both in their aggregation into supramolecular polymers and then their subsequent hierarchical assembly into larger fibre networks often observed in the formation of self-assembled gels. ${ }^{35,36}$ The observed optimum gel strength at a 50:50\% (v/v) ratio of PEG $400:$ water in Fig. 2a coincides with an observed minima for $T_{1}$ relaxation times in PEG 6000 : water, suggesting a sweet-spot for PEG-water interactions at this ratio with little or no "free" or excess PEG available. The observed collapse in $G^{\prime}$ for 1 in PEG 400 : water ratios above $60: 40 \%(\mathrm{v} / \mathrm{v})$ in Fig. 2a may then be explained by the solvation of 1 by the excess PEG 400 that is not interacting with water.

To explore further the role of PEG solvation and why most previous studies did not show any enhancement in gel strength upon on polymer addition, we performed gelation experiments where the order of mixing was varied. Instead of dissolving 1 in PEG, followed by the addition of water (as described above), in these experiments 1 was first dissolved in basic water, followed by the addition of either PEG 400 (Fig. S20, ESI $\dagger$ ), or PEG 400 and glucono- $\delta$-lactone ${ }^{32}$ (Fig. S21, ESI $\dagger$ ). Both methods, with and without glucono- $\delta$-lactone, resulted in gelation; however the addition of glucono- $\delta$-lactone resulted in stronger gels. This shows that while glucono- $\delta$-lactone is not necessary for gelation using this mixing method, it does improve gel strength. Comparing the strength of gels obtained from this mixing method to the gels formed where 1 is dissolved in PEG 400 before the addition of water, we see nearly an order of magnitude difference (2.6-5.6 kPa for gels where 1 is initially dissolved in basic water versus $18 \mathrm{kPa}$ (Fig. 1) for gels where 1 is initially dissolved in PEG 400). In this way, the order of mixing plays a large role in the strength of the hydrogel network.

Macroscopically gels formed when $\mathbf{1}$ was first dissolved in water, followed by PEG 400 addition, are much more opaque than gels formed by 1 when it is first dissolved in PEG 400, followed by water addition (Fig. S22, ESI $\dagger$ ). Visually, a solution of $\mathbf{1}$ in basic ( $\mathrm{pH}$ 9) water is far less transparent than a solution of 1 in PEG 400 (see second vial from right in. Fig. S15, ESI $\dagger$ ), 
suggesting better dissolution is achieved in PEG 400. Combined, these results suggest that the solubility difference of $\mathbf{1}$ in PEG compared to basic water explains why the order of waterPEG mixing is important. This may also explain why previous studies on polymer addition to gels formed from small peptide gelators ${ }^{13-17}$ did in fact show little or even adverse effects on gel strength as they either involved addition of a concentrated DMSO solution to water-polymer mixtures or the addition of gelator-water mixture to the polymer solutions.

\section{Spectroscopy and gel structure}

To investigate the effect of macromolecular crowding and hydrophobic effects on gels formed from 1, a series of attenuated total reflection-infrared spectroscopy (ATR-IR, Fig. 3a and c) and circular dichroism (CD, Fig. 3b and Fig. S23, ESI $\dagger$ ) studies were performed on gels formed from 1 in pure water (or $\mathrm{D}_{2} \mathrm{O}$ ) using aqueous $\mathrm{HCl}$ for the $\mathrm{pH}$ switch method (to avoid interference from glucono- $\delta$-lactone) and the spectra then compared to those obtained from gels formed in 50:50\% (v/v) ethylene glycol or PEG 400 with water (or $\mathrm{D}_{2} \mathrm{O}$ ).

Interestingly, both the ATR-IR and CD data for the ethylene glycol and PEG 400-based gels suggests a significant structural change compared to gels from 1 in water. Deconvolution of the Amide I peaks in the spectra (Fig. 3c) obtained for these gels using $\mathrm{D}_{2} \mathrm{O}$ as a cosolvent, ${ }^{37}$ clearly indicates that the main absorption is around $1651 \mathrm{~cm}^{-1}$. This is close to Byler and Susi's assignment of a band at $1653 \mathrm{~cm}^{-1}$ as arising from $\alpha$-helices in proteins ${ }^{37,38}$ and is not far from their assignment of "unordered" amides around $1645 \mathrm{~cm}^{-1} \cdot{ }^{37}$ It should also be noted that while the peak around $1685 \mathrm{~cm}^{-1}$ matches well up with intermolecular $\beta$-sheets in proteins, ${ }^{37,38}$ recent studies involving isotopic labelling have conclusively shown that in 1 it belongs to the stacked carbamate (Fmoc). ${ }^{39}$ Whilst the peak in the ATR-IR observed at $1651 \mathrm{~cm}^{-1}$ is somewhat similar to that observed by Gazit at $1658 \mathrm{~cm}^{-1}, 31$ the structure of the CD (shown with arrows in Fig. 3 b) for PEG 400 : water hydrogels of 1 is very different, and more related to $\alpha$-helices in proteins. Recent work on other peptide gelators does, however, suggest that the CD spectra might also correspond to other forms of secondary structures, ${ }^{40,41}$ including unordered ones. In contrast, the ATR-IR and CD spectra for gels of formed by $\mathrm{pH}$ switch for 1 do not show any significant unordered or $\alpha$-helical character and appear similar to previously published data on 1 that has been assigned to have $\beta$-sheet like character.

Combined, the results here appear to show a significant unordered or possibly $\alpha$-helical character in the self-assembled structure that 1 forms in PEG: water mixtures. The $\alpha$-helical motif has previously not been identified as major structural feature in peptide-based hydrogels and would only be possible if 1 can form a linear (head-to-tail) spiral assembly, suggesting the unordered secondary structure as a more plausible option. These results also suggest that hydrophobic interactions are as at least as important as macromolecular crowding in the above system as the ATR-IR and CD results are nearly indistinguishable for gels formed in ethylene glycol:water versus PEG 400 : water mixtures. a)

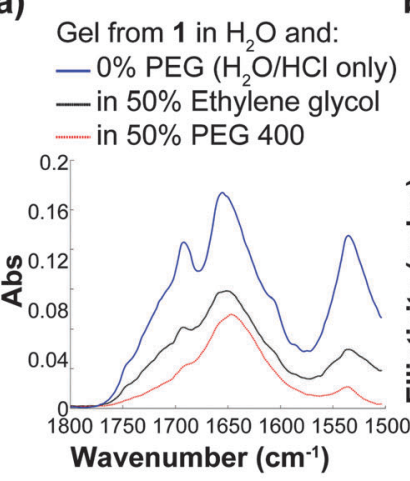

b)

Gel from 1 in $\mathrm{H}_{2} \mathrm{O}$ and: $-0 \% \mathrm{PEG}\left(\mathrm{H}_{2} \mathrm{O} / \mathrm{HCl}\right.$ only) - in $50 \%$ Ethylene glycol

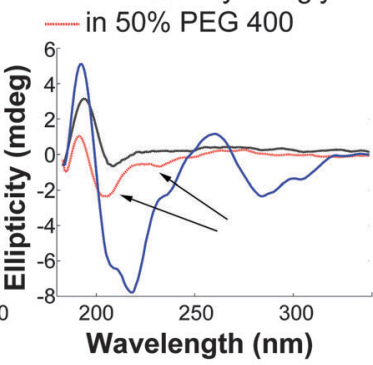

c)

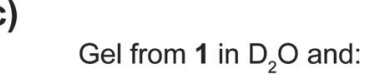

- $0 \% \mathrm{PEG}\left(\mathrm{D}_{2} \mathrm{O} / \mathrm{HCl}\right.$ only)

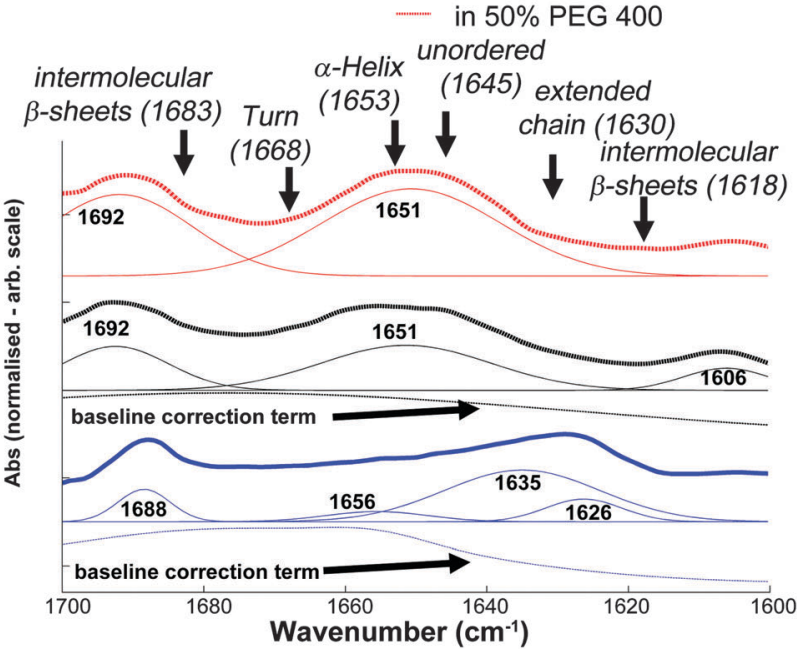

Fig. 3 Spectroscopic studies on the secondary structure of gels formed from 1 in three types of solvents: pure water with the $\mathrm{pH}$ switch method using $\mathrm{HCl}$ (blue), $50: 50 \%(\mathrm{v} / \mathrm{v})$ ethylene glycol : water (black) and $50: 50 \%(\mathrm{v} / \mathrm{v}) \mathrm{PEG}$ 400 : water (red). In (a) and (b) the water is $\mathrm{H}_{2} \mathrm{O}$ while in (c) $\mathrm{D}_{2} \mathrm{O}$ is used (to suppress the Amide II peak around $1550 \mathrm{~cm}^{-1}$ ). ${ }^{29}$ (a) ATR-IR spectra of $1 \%(\mathrm{w} / \mathrm{v})$ hydrogels composed of 1 . (b) CD spectra of $1 \%(\mathrm{w} / \mathrm{v})$ hydrogel of 1 dispersed in water to achieve a final concentration of $0.13 \%(\mathrm{w} / \mathrm{v})$. The arrow highlights the "double negative" peak in the PEG 400 : water spectra (red) around 205-230 $\mathrm{nm}$ that is often associated with $\alpha$-helix structure in proteins. (c) ATR-IR spectra of the Amide I peak region $\left(1600-1700 \mathrm{~cm}^{-1}\right.$ ). The deconvoluted absorption bands (thin lines below thick lines from the measured spectra) with the corresponding absorption maxima were obtained from curve fitting assuming Gaussian band shapes. ${ }^{37}$ The assignments of the components in the Amide I band based on a range of protein structures (in $\mathrm{D}_{2} \mathrm{O}$ ) according to Byler and Susi ${ }^{37,38}$ is also shown with arrows above the top spectrum in (c).

\section{Drug release}

The PEG: water approach outlined here to form peptide-based gels has the additional advantage that a much larger variety of compounds can be co-dissolved and potentially encapsulated in these gels than for traditional hydrogels formed by the $\mathrm{pH}$ switch methods. This includes poorly water-soluble anti-cancer drugs such as Temozolomide ${ }^{42}$ and Paclitaxel (Taxol ${ }^{\mathbb{R}}$ ). ${ }^{43}$ Additionally, Temozolomide ${ }^{44}$ and Paclitaxel ${ }^{45}$ are both unstable under the type of basic conditions ( $\mathrm{pH} \mathrm{8-9)}$ that are typically used to form hydrogels by the $\mathrm{pH}$ switch method. ${ }^{32}$ Previously, 
Huang and co-workers had used partially enzymatically digested konjac glucomannan (KGM) as a co-polymer solvent with 1 to improve the release properties of docetaxel, however, this was with the gel-drug mixture first being dissolved in DMSO and more importantly, the ultimate loading of the drug being limited its solubility in the aqueous phase to $0.01 \%(\mathrm{w} / \mathrm{v})$ of docetaxel in the gel formed by $1(2 \%, \mathrm{w} / \mathrm{v})$ in KGM:water. ${ }^{15}$

We are able to incorporate both Temozolomide and Paclitaxel at concentrations of $0.1 \%(\mathrm{w} / \mathrm{v})$ into our $1 \%(\mathrm{w} / \mathrm{v}) 1$ in PEG 400 : water hydrogels, by co-dissolving Temozolmide or Paclitaxel and 1 in PEG 400 prior to the addition of water, which then resulted in a homogenous opaque gel. The release data was then fitted to the Korsmeyer-Peppas ${ }^{46}$ model according to eqn (1).

$$
M_{\mathrm{t}} / M_{\infty}=K t^{n}
$$

This model describes release from a polymeric system where $M_{\mathrm{t}} / M_{\infty}$ is fractional drug release (usually as \%), $K$ is a characteristic kinetic constant and $n$ an exponent that characterises the mechanism of release. Eqn (1) assumes a cylindrical release matrix which corresponds to the dimensions of the gels formed from 1 . The raw versus fitted data with the resulting values for $K$ and $n$ is shown in Fig. 4.

According to Korsmeyer and Peppas, ${ }^{46}$ a value for $n$ of less than 0.5 suggests that the overall solution diffusion mechanism is Fickian and as $n=0.40 \pm 0.07$ and $0.26 \pm 0.07$ for Temozolomide and Paclitaxel, respectively, in our system, this appears to be the mechanism for the release of both drugs from PEG 400 : water $(50: 50 \%, \mathrm{v} / \mathrm{v})$ hydrogels formed from $1 \%(\mathrm{w} / \mathrm{v})$ 1. We suggest that both drugs are being slowly released from these hydrogels and diffusing into the aqueous phase due to a concentration gradient which eventually results in total release of the drug molecules from the gel matrix. The results suggest that both drugs are considerably smaller than the pore size of the gel network. It should also be noted in this context that only about $9.5 \%$ of 1 is released from the gel over the $48 \mathrm{~h}$ period.

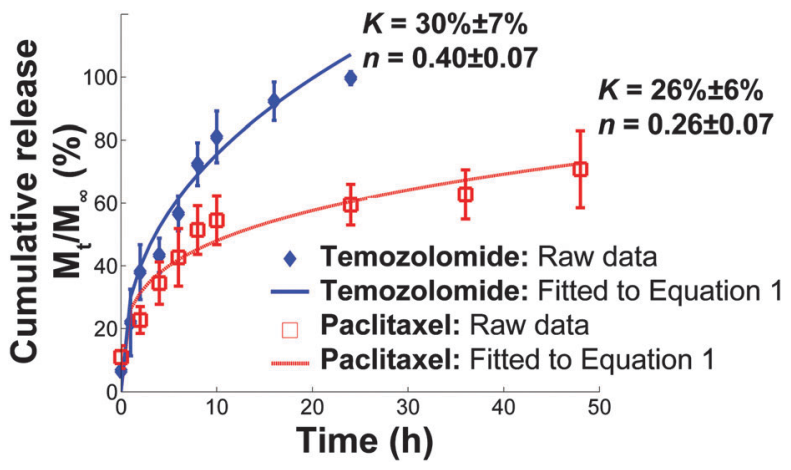

Fig. 4 Cumulative drug release $\left(M_{t} / M_{\infty}, \%\right)$ of $0.1 \%(w / v)$ Temozolomide and Paclitaxel in a 1\% (w/v) gel of 1 in PEG 400 : water using $0.1 \mathrm{M} \mathrm{HCl}+$ $0.5 \%$ Tween 80 as the release media to simulate the gastric environment. Error bars indicate standard deviation from repeat experiments ( $n=3$ for Temozolmide and $n=4$ for Paclitaxel). The fitted release profile according to eqn (1) ${ }^{46}$ is also shown together with the fitted parameters with standard deviation for $n$-repeats.

\section{Conclusions}

We have successfully managed to form hydrogels of 1 using a solvent switch method which employs PEG and water. Spectroscopic studies on these gels suggests a significant secondary structural change when compared to gels formed from pure water. The rheology data does suggest that gels formed in the presence of higher molecular weight PEGs are stronger than those formed with ethylene glycol. This indicates that gelation in these systems is due to a combination of hydrophobic (ethylene glycol) and macromolecular crowding effects with the former probably manifested by the aggregation of 1 into unordered, or even possibly $\alpha$-helical structures, while the macromolecular crowding (excluded volume) effect provides additional gel stability and hence increased gel strength. We hypothesise that the structural change from the typical $\beta$-sheet character to what are probably unordered secondary structures, arises from the stabilisation of intermolecular gelator hydrogen bonds due to the effective dehydration of the gelator in the presence of ethylene glycol and PEGs.

The order of dissolution of $\mathbf{1}$ also appears to play a significant role as gels formed from 1 when it is first dissolved in PEG, followed by water-addition are much stronger than gels first dissolved in water, followed by the addition of PEG. This suggests that the initial complete solubilisation of $\mathbf{1}$ is paramount in controlling the resulting gel properties. This work therefore gives further insight into the factors that govern the formation and properties ${ }^{47}$ of self-assembled hydrogels and in turn may also give insight into the self-assembly and properties of biological gel-like materials such as the cytosol and the extracellular matrix (ECM).

\section{Experimental section}

\section{Materials}

All chemicals including different types of PEG (ethylene glycol Cat\# 102466, PEG 200 - Cat\# P3015, PEG 400 - Cat\# P3265, PEG 8000 - Cat\# 89510, PEG 10000 - Cat\# 81280 and PEG 20000 Cat\# 95172 - all bis-hydroxy terminated) and glucono- $\delta$-lactone were purchased from Sigma Aldrich, and used as received. MilliQ Water (resistivity 18.2 M $\Omega$ ) was used throughout the study. Paclitaxel $\left(\right.$ Taxol $^{\circledR}$ ) was obtained from LC Laboratories USA and Temozolomide from Sigma Aldrich. The solution phase synthesis of $\mathbf{1}$ was adapted from the methods (ESI $\dagger$ ) previously reported by König and Rödela ${ }^{48}$ and Gagnon et al. ${ }^{49}$

\section{Preparation of gels from 1 in PEG/water mixtures (PEG-gelator to water)}

For PEG: $\mathrm{H}_{2} \mathrm{O}$ hydrogels made from ethylene glycol, PEG 200 and PEG 400, 1 was dissolved in the selected PEG at the appropriate target concentration with the aid of sonication to give a transparent solution of $\mathbf{1}$ in PEG. Following this, milliQ water was added to the solution of $\mathbf{1}$ in PEG in order to attain the desired final ratio of PEG to water and the resultant opaque solution shaken by hand for a few seconds to ensure thorough mixing and left to stand at ambient temperature. As PEG 8000, 
PEG 10000 and PEG 20000 are solid at room temperature, they were first melted by heating to $\mathrm{ca} .70{ }^{\circ} \mathrm{C}$ (all three PEGs possess a melting point around $60-70{ }^{\circ} \mathrm{C}$ ). The gelator 1 was then dissolved in the melt, and after a brief period of mixing, water was added to solution of $\mathbf{1}$ in the melt and resulting mixture mixed rapidly by shaking for a few seconds and then left to cool at room temperature. Hydrogels formed on a timescale of seconds to minutes, depending on the PEG: water ratio and the concentration of $\mathbf{1}$. The formation of self-supporting hydrogels was examined through the vial inversion test. For the preparation of PEG: water mixtures without 1, PEG 8000, PEG 10000 and PEG 20000 were also melted first before the addition of the required amount of water whereas ethylene glycol, PEG 200 and PEG 400 were readily miscible with water making addition of water to those compounds straightforward.

\section{Preparation of gels from 1 by dissolving first in water, followed} by a pH switch or PEG addition

For hydrogels formed via $\mathrm{pH}$ switch, 1 was dissolved using a basic aqueous solution at $\mathrm{pH}$, through the addition of one equivalent of aqueous sodium hydroxide $(0.1 \mathrm{M})$. This solution was sonicated to aid in the dissolution of $\mathbf{1}$. Four different approaches were then used to form gels from this mixture:

Glucono- $\delta$-lactone $\mathbf{p H}$ switch $\operatorname{method}^{32}$. For rheological comparison with the PEG: water mixtures (Fig. 1), one equivalent of glucono- $\delta$-lactone was added to a basic solution of $\mathbf{1}$ to gradually lower the $\mathrm{pH}$ of the system, ${ }^{32}$ resulting in the formation of an opaque hydrogel over the course of approximately half an hour. This method gives consistently the strongest hydrogels when using pure water.

Water-gelator to PEG without $\mathbf{p H}$ switch. To investigate the effect of PEG-gelator-water mixing order, PEG 400 was added to the basic solution of 1 above to give a final ratio $50: 50 \%(\mathrm{v} / \mathrm{v})$ of PEG 400:water resulting in the formation of an opaque hydrogel.

Water-gelator to PEG with $\mathbf{p H}$ switch. In an alternative experiment to investigate the effect of PEG-gelator-water mixing order, PEG 400 was added to the basic solution of 1 above to give a final ratio $50: 50 \%(\mathrm{v} / \mathrm{v})$ of PEG 400 : water, followed by the addition of one equivalent of glucono- $\delta$-lactone to gradually lower the $\mathrm{pH}$ of the system, ${ }^{32}$ resulting in the formation of an opaque hydrogel.

HCl pH switch method. For ATR-IR and CD studies (Fig. 3), the use of glucono- $\delta$-lactone was avoided as additional CD signals arising due to its use can interfere with the interpretation of the IR studies. For this reason the $\mathrm{pH}$ of the above basic solution was slowly lowered by the careful addition of dilute aqueous hydrochloric acid $(\approx 0.1 \mathrm{M})$ until an opaque hydrogel had been formed.

\section{Rheology}

Rheological measurements were performed on an Anton Paar MCR 302 rheometer using a $25 \mathrm{~mm}$ stainless steel parallel plate geometry configuration and analysed using RheoPlus v3.61 software. Typical rheology measurements involved casting $600 \mu \mathrm{L}$ of a solution of interest onto one of the stainless steel plates, lowering the other plate to the measurement position $(1 \mathrm{~mm}$ gap), and allowing an hour for the gel to form via the PEG to water solvent switch as described above. Frequency sweeps were performed with a $\log$ ramp frequency $(f)=0.01-10 \mathrm{~Hz}$ and constant strain $(\gamma=0.5 \%)$. Amplitude Sweeps were performed at a constant frequency $(f)=1 \mathrm{~Hz}$ and log ramp strain $(\gamma=0.1-100 \%)$. A Peltier temperature control hood was used to maintain a temperature of $25{ }^{\circ} \mathrm{C}$ for frequency and amplitude sweeps. The rheology plots displayed are an average of at least three repeats for each point and error bars denote two standard deviations from the log-averaged mean.

\section{CD measurements}

CD measurements were performed using a ChirascanPlus CD spectrometer, with data collected between wavelengths of 180-500 nm with a bandwidth of $1 \mathrm{~nm}$, sample ratio of $0.1 \mathrm{~s}$ per point and step of $1 \mathrm{~nm}$. In a typical experiment, $1 \%(\mathrm{w} / \mathrm{v})$ hydrogels were prepared and dispersed in water using a $1: 8(\mathrm{v} / \mathrm{v})$ dilution ( $c a .0 .125 \% 1(\mathrm{w} / \mathrm{v})$ ). Temperature was kept constant at $20{ }^{\circ} \mathrm{C}$ and all experiments were repeated three times and averaged into a single plot. The resulting spectra were smoothed using the Savitzky-Golay smoothing function sgolayfilt (7th order, frame size $=41)$ in matlab.

\section{ATR-IR measurements}

Attenuated total reflectance infrared spectroscopy (ATR-IR) measurements were made on a Perkin Elmer Spotlight 400 FT-IR spectrophotometer equipped with a diamond crystal attenuated total reflectance (ATR) accessory. Hydrogels were prepared with $1 \%(\mathrm{w} / \mathrm{v}) \mathrm{I}$ using either $\mathrm{D}_{2} \mathrm{O}$ or $\mathrm{H}_{2} \mathrm{O}$ for the HCl-based $\mathrm{pH}$ switch method or in 50:50\% (v/v) mixtures of ethylene glycol or PEG 400 with either $\mathrm{D}_{2} \mathrm{O}$ or $\mathrm{H}_{2} \mathrm{O}$ as appropriate and pressed between the diamond crystal and substrate. All spectra were scanned 16 times over the range of $4000-650 \mathrm{~cm}^{-1}$.

Deconvolution of the ATR-IR spectra of $1 \%(\mathrm{w} / \mathrm{v})$ of 1 , recorded in either pure $\mathrm{D}_{2} \mathrm{O}$ (to suppress interference in the Amide I region from $\left.\mathrm{H}_{2} \mathrm{O}\right)^{37}$ with the HCl-based $\mathrm{pH}$ switch method to trigger gelation or in 50:50\% (v/v) mixtures of ethylene glycol or PEG with $\mathrm{D}_{2} \mathrm{O}$, was carried out using the approach outlined by Byler and Susi. ${ }^{37}$ Briefly, only the region of each spectra between $1600-1700 \mathrm{~cm}^{-1}$ was fitted to multiple Gaussians. The fitting process was carried out using the curve fitting tool in matlab and the number of Gaussians increased until a fit with a $R^{2}>0.99$ was achieved while restricting the amplitute of the Gaussian peaks to be non-negative. The normalised spectra were then plotted (Fig. 3) and the maximum of each peak determined from the fitted Gaussian models. In the case of $\mathbf{1}$ in pure $\mathrm{D}_{2} \mathrm{O}$ with $\mathrm{HCl}$ and $\mathbf{1}$ with ethylene glycol:water $(50: 50 \%, \mathrm{v} / \mathrm{v})$, an additional broad-Gaussian baseline correction term was also included.

\section{Temozolomide and Paclitaxel release study}

To prepare a hydrogel for drug release studies, $1 \mathrm{mg}$ of Temozolomide or $1 \mathrm{mg}$ of Paclitaxel $\left(\right.$ Taxol $^{\mathbb{R}}$ ) was dissolved in $500 \mu \mathrm{L}$ of the selected PEG, followed by the addition of $10 \mathrm{mg} \mathbf{1}$. This mixture was then sonicated, resulting in a clear solution. 
$500 \mu \mathrm{L}$ of milliQ water was then added, giving a final concentration of $50 \%$ PEG (v/v) and $1 \% 1(\mathrm{w} / \mathrm{v})$. As above, the addition of water results in the gelation of the sample, and this gel was left to stand overnight.

For release studies, release media $(0.1 \mathrm{M}$ aqueous hydrochloric acid and $0.5 \%$ Tween 80 ) was added on top of the hydrogel, and an aliquot $(50 \mu \mathrm{L})$ removed for analysis at different time points. After each sampling point, the release media was replenished with an equal volume of fresh media to what was removed. Analysis of the release was performed using a Shimadzu UFLC (Model LC-20AD). For analysis of Temozolomide the mobile phases consisted of a $10 \mathrm{mM}$ ammonium phosphate buffer at pH 3.25 and methanol, respectively, mixed in an 88:12 ratio. An XBridge ${ }^{\mathrm{TM}} \mathrm{C} 18$ column was utilised, an isocratic elution method with a flow rate of $1 \mathrm{~mL} \min ^{-1}$ used and absorbance measured at $254 \mathrm{~nm}$ with Temozolomide eluting at $t_{\mathrm{R}}=4 \mathrm{~min}$. For analysis of Paclitaxel the mobile phase was prepared from a degassed mixture of water and acetonitrile (55:45 v/v). An XBridge $^{\mathrm{TM}}$ C18 column was used, monitoring the absorbance at $227 \mathrm{~nm}$ using an isocratic flow of $1.0 \mathrm{~mL} \mathrm{~min}^{-1}$ with Paclitaxel eluting at $t_{\mathrm{R}}=7.0 \mathrm{~min}$.

Temozolomide and Paclitaxel samples with known concentrations without the gelator were used to create a calibration curve and from this, the concentrations of unknown samples were determined. LC Solutions (version 3.60) software was used to determine the areas of unknown samples and from these; the quantities of drug released at different time intervals were calculated. The release profiles were then fitted to the Korsmeyer-Peppas model (Eqn (1)) ${ }^{46}$ using the unrestrained non-linear regression (Levenberg-Marquart) nlinfit function in matlab.

\section{Acknowledgements}

This research is supported by the Australian Research Council (ARC) Discovery Project Grant (DP130101512), ARC Centre of Excellence Grant (CE140100036), ARC Future Fellowship to PT (FT120100101) and the Australian Government by PhD Scholarship to MdMH.

\section{Notes and references}

1 R. J. Ellis, Trends Biochem. Sci., 2001, 26, 597.

2 C. A. Strulson, R. C. Molden, C. D. Keating and P. C. Bevilacqua, Nat. Chem., 2012, 4, 941.

3 G. W. Li, O. G. Berg and J. Elf, Nat. Phys., 2009, 5, 294.

4 M. S. Cheung, D. Klimov and D. Thirumalai, Proc. Natl. Acad. Sci. U. S. A., 2005, 102, 4753.

5 D. D. L. Minh, C. E. Chang, J. Trylska, V. Tozzini and J. A. McCammon, J. Am. Chem. Soc., 2006, 128, 6006.

6 A. S. Zeiger, F. C. Loe, R. Li, M. Raghunath and K. J. Van Vliet, PLoS One, 2012, 7, e37904.

7 P. Kumar, A. Satyam, X.-L. Fan, E. Collin, Y. Rochev, B. J. Rodriguez, A. Gorelov, S. Dillon, L. Joshi, M. Raghunath,
A. Pandit, D. I. Zeugolis and I. Dimitrios, Sci. Rep., 2015, 5, 8729.

8 Y. Q. Wang, M. Sarkar, A. E. Smith, A. S. Krois and G. J. Pielak, J. Am. Chem. Soc., 2012, 134, 16614.

9 L. A. Benton, A. E. Smith, G. B. Young and G. J. Pielak, Biochemistry, 2012, 51, 9773.

10 M. Senske, L. Tork, B. Born, M. Havenith, C. Herrmann and S. J. Ebbinghaus, J. Am. Chem. Soc., 2014, 136, 9036.

11 D. Gnutt, M. Gao, O. Brylski, M. Heyden and S. Ebbinghaus, Angew. Chem., Int. Ed., 2015, 54, 2548.

12 N. Yan, Z. Xu, K. K. Diehn, S. R. Raghavan, Y. Fang and R. G. Weiss, J. Am. Chem. Soc., 2013, 135, 8989.

13 J. Wang, Z. Wang, J. Gao, L. Wang, Z. Yang, D. Kong and Z. Yang, J. Mater. Chem., 2009, 19, 7892.

14 L. Chen, S. Revel, K. Morris, D. G. Spiller, L. C. Serpell and D. J. Adams, Chem. Commun., 2010, 46, 6738.

15 R. Huang, W. Qi, W. Feng, R. Su and Z. He, Soft Matter, 2011, 7, 6222.

16 G. Pont, L. Chen, D. G. Spiller and D. J. Adams, Soft Matter, 2012, 8, 7797.

17 D. J. Cornwell and D. K. Smith, Mater. Horiz., 2015, 2, 279. 18 M. Zhou, A. M. Smith, A. K. Das, N. W. Hodson, R. F. Collins, R. V. Ulijn and J. E. Gough, Biomaterials, 2009, 30, 2523.

19 W. T. Truong, Y. Su, J. T. Meijer, P. Thordarson and F. Braet, Chem. - Asian J., 2011, 6, 30.

20 W. T. Truong, L. Lewis and P. Thordarson, in Functional Molecular Gels, ed. J. F. Miravet and B. Escuder Gillier, Royal Society of Chemistry, Cambridge, UK, 2014, ch. 6, pp. 156-194.

21 Y. Nagai, H. Yokoi, K. Kaihara and K. Naruse, Biomaterials, 2012, 33, 1044.

22 X.-Q. Dou, X.-M. Yang, P. Li, Z.-G. Zhang, H. Schönherr, D. Zhang and C.-L. Feng, Soft Matter, 2012, 8, 9539.

23 A. Friggeri, B. L. Feringa and J. van Esch, J. Controlled Release, 2004, 97, 241.

24 M. L. Briuglia, A. J. Urquhart and D. A. Lamprou, Int. J. Pharm., 2014, 474, 103.

25 J. T. King, E. J. Arthur, C. L. Brooks III and K. J. Kubarych, J. Am. Chem. Soc., 2014, 136, 188.

26 D. Miyoshi, H. Karimata and N. J. Sugimoto, J. Am. Chem. Soc., 2006, 128, 7957.

27 C. B. Stanley and H. B. Strey, Biophys. J., 2008, 94, 4427.

28 A. Roque, I. Ponte and P. Soau, Biophys. J., 2007, 93, 2170.

29 M. Reches and E. Gazit, Isr. J. Chem., 2005, 45, 363.

30 V. Jayawarna, M. Ali, T. A. Jowitt, A. F. Miller, A. Saiani, J. E. Gough and R. V. Ulijn, Adv. Mater., 2006, 18, 611.

31 A. Mahler, M. Reches, M. Rechter, S. Cohen and E. Gazit, Adv. Mater., 2006, 18, 1365.

32 D. J. Adams, M. F. Butler, W. J. Frith, M. Kirkland, L. Mullen and P. Sanderson, Soft Matter, 2009, 5, 1856.

33 S. J. Hermansky, D. A. Neptun, K. A. Loughran and H. W. Leung, Food Chem. Toxicol., 1996, 33, 139.

34 E. M. Clop, M. A. Perillo and A. K. Chattah, J. Phys. Chem. B, 2012, 116, 11953.

35 L. A. Estroff and A. D. Hamilton, Chem. Rev., 2004, 104, 1201. 
36 K. W. T. Tong, S. Dehn, J. E. A. Webb, K. Nakamura, F. Braet and P. Thordarson, Langmuir, 2009, 25, 8586.

37 D. M. Byler and H. Susi, Biopolymers, 1986, 25, 469.

38 F. Meersman, L. Smeller and K. Heremans, Biophys. J., 2002, 82, 2635.

39 S. Fleming, P. W. J. M. Frederix, I. Ramos-Sasselli, N. Hunt, R. V. Ulijn and T. Tuttle, Langmuir, 2013, 29, 9510.

40 X. Mu, K. M. Eckes, M. M. Nguyen, L. J. Suggs and P. Ren, Biomacromolecules, 2012, 13, 3562.

41 K. L. Morris, L. Chen, A. Rodger, D. J. Adams and L. C. Serpelli, Soft Matter, 2015, 11, 1174.

42 M. F. G. Stevens, J. A. Hickman, R. Stone, N. W. Gibson, G. U. Baig, E. Lunt and C. G. Newton, J. Med. Chem., 1984, 27, 196.
43 M. C. Wani, H. L. Taylor, M. E. Wall and P. Coggon A. T. McPhail, J. Am. Chem. Soc., 1971, 93, 2325.

44 E. S. Newsland, M. F. G. Stevens, S. R. Wedge, R. T. Wheelhouse and C. Brock, Cancer Treat. Rev., 1997, 23, 35.

45 S. L. Richheimer, D. M. Tinnermeier and D. W. Timmons, Anal. Chem., 1992, 64, 2323.

46 R. W. Korsmeyer, R. Gurny, E. Doelker, P. Buri and N. A. Peppas, Int. J. Pharm., 1983, 15, 25.

47 J. H. van Esch, Langmuir, 2009, 25, 8392.

48 B. König and M. Rödela, Synth. Commun., 1999, 29, 943.

49 P. Gagnon, X. Huang, E. Therrien and J. W. Keillor, Tetrahedron Lett., 2002, 43, 7717. 